\title{
Model Predictive Control for Energy and Leakage Management in Water Distribution Systems *
}

\author{
Piotr Skworcow* Bogumil Ulanicki ${ }^{*}$ Hossam AbdelMeguid* \\ Daniel Paluszczyszyn * \\ * Process Control - Water Software System, De Montfort University \\ Leicester, UK (e-mails: \{pskworcow, bul, habdelmeguid, \\ dpaluszczyszyn\}@dmu.ac.uk)
}

\begin{abstract}
This paper considers development of a methodology for an on-line energy and leakage management in water distribution systems, formulated within a model predictive control framework. The approach involves calculation of control actions, i.e. time schedules for pumps, valves and sources, to minimize the costs associated with energy used for water pumping and treatment and water losses due to leakage, whilst satisfying all operational constraints. The process of computing the control action utilises EPAnet hydraulic simulator, a mathematical modelling language called GAMS and a non-linear programming solver called CONOPT. The proposed control scheme has been integrated with an industrial SCADA system from ABB and interfaced with an actual medium-scale water distribution systems being part of Yorkshire Water Services. The scheme is currently being tested using on-line telemetry data. It has been operational for over 1 month with 1 hour sampling time and the preliminary results described in this paper indicate a potential for savings of $30 \%$ of the cost of electrical energy.
\end{abstract}

Keywords: Model predictive control, water distribution systems, pump control, SCADA, nonlinear optimisation

\section{INTRODUCTION}

Water distribution systems (WDS), despite operational improvements introduced over the last 10-15 years, still lose a considerable amount of potable water from their networks due to leakage, whilst using a significant amount of energy for water treatment and pumping. Reduction of leakage, hence savings of clean water, can be achieved by introducing pressure control algorithms (Ulanicki et al., 2000). Amount of energy used for pumping can be decreased through optimisation of pumps operation (Ormsbee and Lansey, 2007; Bounds et al., 2006). An approach to combine energy and leakage optimisation was recently reported in (Skworcow et al., 2009b).

Optimisation studies for medium or large-scale WDS are typically carried out off-line. The main purpose of commercial optimisation packages, such as $\mathrm{H}_{2} O N E T$ from MWH Soft or Aquis from 7-Technologies, is to pinpoint the most cost-effective operational policies for a specific water system. There exists an on-line optimisation package called Derceto (Bunn, 2007), but it is highly specialised and uses linearised models built purposely for a particular network. This means that any changes to the water network require significant changes in the optimisation model, which leads to high cost of system maintenance.

This paper considers development of a methodology for online energy and leakage management in WDS formulated

\footnotetext{
* This research is sponsored by and is a part of EPSRC Neptune
} project (www.neptune.ac.uk) within model predictive control (MPC) framework. MPC has been applied in wide range of industrial applications, see e.g. (Morari and Lee, 1999; Qin and Badgwell, 2003), including open water systems (Igreja and Lemos, 2009; Overloop, 2006). Application of MPC for pump management in WDS was proposed in (Biscos et al., 2003) but that study considered only off-line simulations and some abstract small-scale case study. This paper presents an online implementation of MPC integrated with an industrial supervisory control and data acquisition (SCADA) system and interfaced with an actual medium-scale WDS.

The paper is structured as follows. In Section 2 the problem of energy and leakage management in WDS is formulated. Section 3 describes formulation and development of a model predictive controller. The case study and the results obtained to date are discussed in Section 4. Conclusions are given in Section 5.

\section{PROBLEM FORMULATION}

The cost of electrical energy used for water treatment and pumping is a major factor contributing to the operational cost of WDS (Ulanicki et al., 2007). Typically operation of pump stations in WDS is governed by some local control loops; number of pumps switched on and their speed (if the pump station is equipped with variable speed drive) usually depends on levels of reservoirs. Such approach, whilst being simple and robust, often leads to intensive pumping during the expensive electricity tariff period which typically coincides with the periods of high demand 
for water. In many WDS the pumps can also be operated remotely from a control room: manually by an operator or automatically according to time schedules.

Commercial optimisation packages can tune off-line local control loops or calculate pump schedules to minimise some criteria, with the main goal being minimisation of the operational cost. Such optimisation uses models of WDS and is not a trivial task: WDS exhibit highly nonlinear characteristics, there is a number of operational constraints (described in Section 3.4), set of control variables contains both discrete (e.g. number of pumps 'on') and continuous (e.g. pump speed) variables and can be of significant size, particularly for large-scale systems.

The approach considered in this paper involves calculation of control actions, i.e. time schedules for pumps, valves and sources, to minimize the costs associated with energy used for water pumping and treatment and water losses due to leakage. The schedules should be calculated over some time horizon which corresponds to a cyclic behaviour of demand patterns, e.g. 24 hours or 1 week. Furthermore, it is desired to calculate the schedules on-line, taking into account current state of the system and to re-calculate the schedules periodically in order to account for unpredicted changes in system state, due to demands variations and modelling uncertainty. Hence, it seems natural to formulate a control strategy within MPC framework.

\section{DEVELOPMENT OF MODEL PREDICTIVE CONTROLLER}

Model predictive control (MPC) is not a specific control strategy, but rather an ample range of control methods developed around three common ideas (Camacho and Bordons, 2004): (i) the explicit use of a model to predict the process response at future time instants, (ii) the calculation of a control sequence by minimizing a certain cost function and (iii) the use of a receding horizon strategy. The following subsections describe a WDS modelling approach, an overall control scheme, constraints, a cost function and implementation of an optimisation process.

\subsection{Water distribution system model}

A hydraulic model of WDS consists of: boundary conditions (sources and exports), a hydraulic nonlinear network made up of pipes, pumps and valves, and reservoir dynamics. Each network component has a hydraulic equation and WDS can be described by differential-algebraic equations (DAE), where the differential part corresponds to reservoir dynamics and the algebraic part corresponds to network equations (Brdys and Ulanicki, 1994). In this work discrete-time models are used.

A large-scale WDS can have thousands to tens of thousand of nodes and pipes. For model-based control purposes, in order to reduce the computational burden, the full hydraulic model is simplified using model reduction algorithm. In a simplified model all control elements remain unchanged, but the number of pipes and nodes is significantly reduced. The algorithm involves linearisation, Gaussian elimination, and reconstruction of a simplified nonlinear model; see (Ulanicki et al., 1996) for details.

\subsection{Overall control scheme}

An overall control scheme is illustrated in Figure 1 and each of its elements is described below. Note that the notation $u(k+i \mid k)$ denotes value of $u$ at time $k+i$ calculated at time $k$.

Operator specifies constraints and boundary conditions based on some global operational policies. Inclusion of model reduction module enables adaptation to abnormal situations and structural changes in a network, e.g. isolation of part of a network due to pipe burst. In such case an operator can change the full hydraulic model and run model reduction module to automatically produce updated simplified model. In normal operation a simplified model is fixed.

The role of SCADA system is to apply an immediate control $u(k \mid k)$ into WDS and to provide readings from telemetry: flows $q$ and pressures $r$ across the network. Vector $u(k \mid k)$ consists of setpoints for: pump stations (number of pumps 'on' denoted $n$ and, for pumps equipped with variable speed drive, their speed denoted $s$ ), sources (production at water treatment works) and valves (depends on type of valve). These elements of WDS are then operated by local controllers according to the setpoints specified in SCADA. Note that in practice the operation of some network elements may be specified by an operator as 'fixed' and thus cannot be controlled by MPC.

Demand prediction module reads demands history $\{d(k-$ $\left.\left.H_{d} \mid k\right) \ldots d(k \mid k)\right\}$ (using selected past flow measurements) and calculates predicted demands $\left\{\hat{d}(k+1 \mid k) \ldots \hat{d}\left(k+H_{p}-\right.\right.$ $1 \mid k)\}$ which, together with the most recent measurements $d(k \mid k)$, are fed into MPC. $H_{p}$ denotes prediction horizon and $H_{d}$ denotes length of demands history. In this work $H_{d}=6$ weeks and the demand prediction algorithm used is based on thresholding, filtering and exponential smoothing; see (Rance et al., 2001) for details.

At every time instant $k$ (typically every 1 hour) MPC reads all required signals illustrated in Figure 1, calculates control schedule $\left\{u(k \mid k) \ldots u\left(k+H_{p}-1 \mid k\right)\right\}$ and writes it to SCADA. Since the dynamics in a WDS model described by DAE are associated with reservoir pressures, a set of current reservoir pressures $r(k \mid k)$ retrieved from SCADA can be considered as a state-feedback, while the boundary conditions together with the predicted demands can be considered as a reference to be tracked.

\subsection{Cost function}

The objective function to be minimised is the total energy cost for water treatment and pumping. Such formulation, combined with the use of a network model with pressure-depended leakage, was demonstrated to lead to a simultaneous minimisation of energy usage and water losses (Skworcow et al., 2009a). Pumping cost depends on pumps power consumption and on the electricity tariff. The tariff is usually a function of time with cheaper and more expensive periods. For given sampling time $\tau$, the objective function $V(k)$ is given by the following equation: 


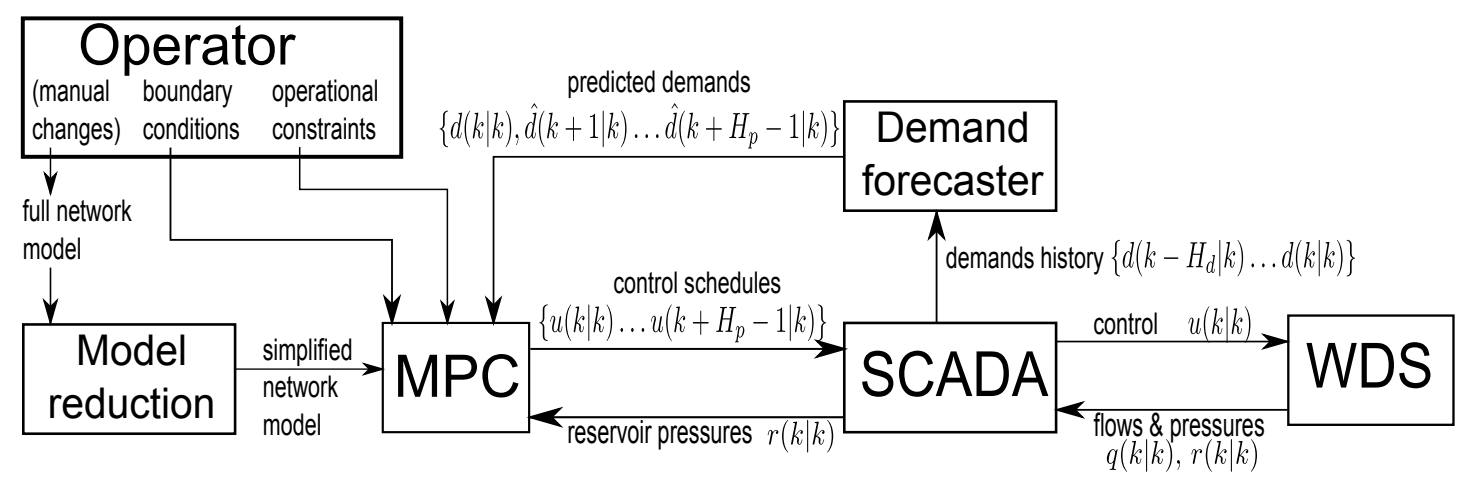

Fig. 1. Overall control scheme linked to WDS via SCADA system.

$$
\begin{aligned}
V(k) & =\left(\sum_{j \in J_{p}} \sum_{i=k}^{k+H_{p}-1} \gamma_{p}^{j}(i) f_{j}\left(q^{j}(i), n^{j}(i), s^{j}(i)\right)\right. \\
& \left.+\sum_{j \in J_{s}} \sum_{i=k}^{k+H_{p}-1} \gamma_{s}^{j}(i) q_{s}^{j}(i)\right) \tau
\end{aligned}
$$

where $J_{p}$ is the set of indices for pump stations and $J_{s}$ is the set of indices for treatment works. The function $\gamma_{p}^{j}(i)$ represents the electrical tariff. The treatment cost for each water treatment works is proportional to the flow output with the unit price of $\gamma_{s}^{j}(i)$. The term $f_{j}\left(q^{j}(i), n^{j}(i), s^{j}(i)\right)$ represents the electrical power consumed by pump station $j$, where $n^{j}(i)$ denotes number of pumps 'on' and $s^{j}(i)$ denotes their normalised speed, with $s^{j}(i)=1$ for fixed speed pumps. For details on modelling of pump power consumption and the form of function $f_{j}$ see (Ulanicki et al., 2008).

\subsection{Constraints}

The fundamental requirement during optimisation is that all calculated variables satisfy the hydraulic model equations; non-linear network equations play the role of equality constraints.

In addition to the equality constraints, operational constraints are applied to keep the system state within its feasible range. The typical requirements of network scheduling are concerned with reservoir levels in order to prevent emptying or overflowing, and to maintain adequate storage for emergency purposes. Similar constraints must be applied to the pressures at critical connection nodes, since water utilities are obliged by the regulatory bodies to maintain required pressures throughout the water network. Another constraint is on the final water level of reservoirs, such that the final level (at time $k+H p-1$ ) is not smaller than the initial level (at time $k$ ). The control variables such as the number of pumps switched on, pump speeds or valve positions, are also constrained by lower and upper constraints determined by the features of the control components.

\subsection{Implementation}

In the optimisation problem considered some of the decision variables are continuous (e.g. water production, pump speed, valve position) and some are integer (e.g. number of pumps switched on). Such mixed-integer problems and are hard to solve numerically even for medium-scale models. Continuous relaxation of integer variables (e.g. allowing 2.5 pumps on) enables network scheduling to be treated initially as a continuous optimisation problem solved by a non-linear programming algorithm. Subsequently, the continuous solution is transformed into an integer solution (integer number of pumps 'on') by further automatic post-processing. Note that such two-stages approach was reported in e.g. (Bounds et al., 2006; Ulanicki et al., 2007; Skworcow et al., 2009a). In this work the post-processing algorithm to generate an integer solution allows up to two on/off switches during a specified time interval (typically 1 to 3 hours). The integer solution is such that the average flow of each pump station in every time interval matches the flow of a continuous solution. Such post-processing approach increases the cost typically by 1 to $3 \%$ compared to the continuous solution cost.

MPC implementation scheme is illustrated in Figure 2. The main control module was implemented in $\mathrm{C \#}$ and its operation at each $k$ is as follows:

(1) Load simplified network model, update the model using "live" signals: predicted demands, reservoir pressures and boundary conditions.

(2) Simulate the model using an open-source hydraulic simulator called EPAnet (http://www.epa.gov) and retrieve simulation results.

(3) Using simplified hydraulic model, simulation results and constraints signals generate continuous optimisation problem written in a mathematical modelling language called GAMS (Brooke et al., 1998), which calls up a commercial non-linear programming solver called CONOPT (Drud, 1985).

(4) Process results from CONOPT to generate an integer solution. An integer solution constitutes the control schedules which are subsequently written to SCADA.

In non-linear programming initial conditions plays an important role. Well-posed initial conditions can speed up the convergence, while initial conditions far from a feasible region may result in a solver being unable to find even a feasible solution. For this reason a hydraulic simulator is used to provide such initial condition, where at least the equality constraints (network equations) are satisfied. Furthermore, the hydraulic model is updated using the most recent reservoir pressure measurements $r(k \mid k)$ and the previously calculated control schedule $\{u(k-1 \mid k-$ 1) $\left.\ldots u\left(k+H_{p}-2 \mid k-1\right)\right\}$, so the initial condition should be in a feasible region and could even be close to the optimum. 


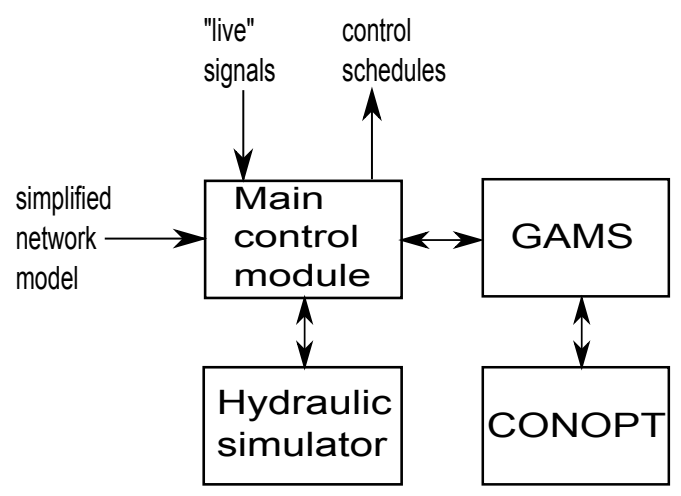

Fig. 2. MPC implementation scheme.

Such approach is possible due to using a receding horizon strategy.

Note that, if the time of control schedules computation was significant compared to $\tau$, then the formulas given in previous subsections would need to be reformulated to calculate $\left\{u(k+1 \mid k) \ldots u\left(k+H_{p} \mid k\right)\right\}$ instead of $\left\{u(k \mid k) \ldots u\left(k+H_{p}-\right.\right.$ $1 \mid k)\}$. Similarly, in practical applications the telemetry together with SCADA systems may introduce a delay which, if significant, needs to be taken into account.

\section{CASE STUDY}

The proposed MPC has been developed, implementation of an overall control scheme (illustrated in Figure 1) is being finalised and the overall scheme is currently being tested using live data from an actual WDS. In this section preliminary results of these test are described.

\subsection{Water distribution system}

The WDS considered is a medium scale network, being part of Yorkshire Water Services (YWS). The network model provided by YWS consists of 2074 nodes, 2212 pipes, 8 pumps in 5 pump stations, 4 valves which potentially could be controlled and 4 reservoirs. Average total demand including exports is approximately 400 to $500 \mathrm{l} / \mathrm{s}$, depending on season. Schematic of the case study network is illustrated in Figure 3. Note that the provided network model has been developed and calibrated by YWS. All imports are treated as boundary conditions and are not included in the control vector. For further details about this WDS see (Skworcow et al., 2009a).

There were no structural changes in the simplified model WDS and the operational constraints, formulated via discussions with YWS personnel, were fixed during the on-line tests carried out to date. However, there were some engineered events carried out by YWS, namely simulation of pipe bursts via opening of fire hydrants, in order to test the MPC and also other systems developed by project partners, in a wider envelope of operating conditions.

\subsection{SCADA system}

The WDS considered is linked to an industrial SCADA system from ABB (www.abb.com) called 800xA, originally developed for power generation systems. Developed software modules constituting the implementation illustrated

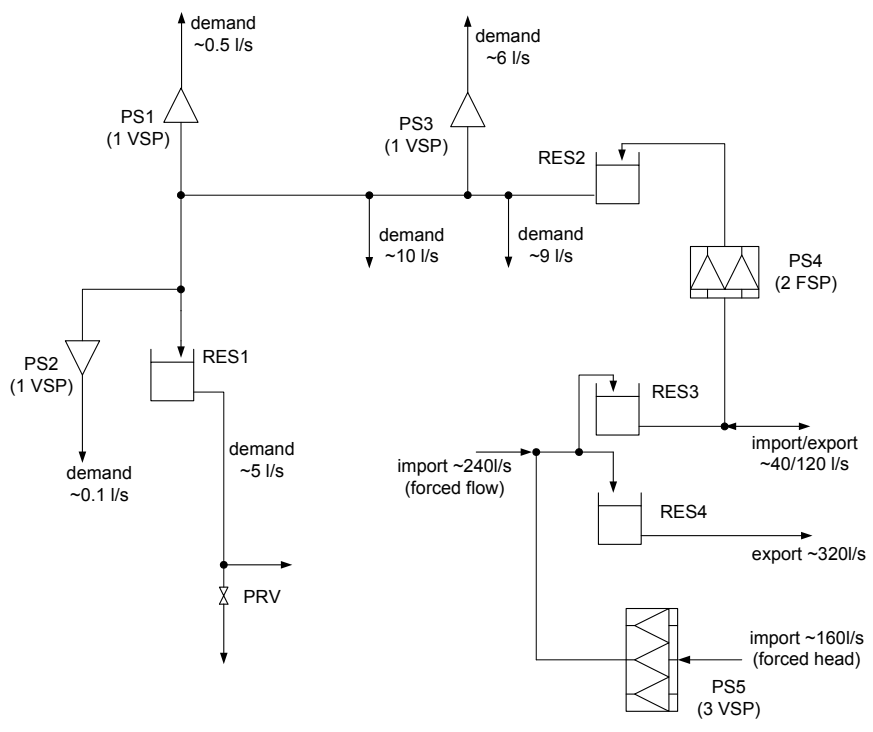

Fig. 3. Structure of the YWS case study network. Abbreviations denote: PS - pumping station, FSP - fixed speed pump, VSP - variable speed pump, RES reservoir. Demands and average import/exports are also depicted.

in Figure 2 were installed onto a server provided by $\mathrm{ABB}$, which runs $800 x \mathrm{x}$ platform in parallel. This allows to retrieve all required "live" pressure and flow data from the WDS and to write the control schedules with minimal delay. All measurements and control signals can be stored in $800 \mathrm{xA}$ for several years.

In current implementation the control actions calculated by MPC are not actually applied in the WDS due to a lack of required remote-control infrastructure in YWS system. Calculated control schedules can, however, be displayed by an operator in a graphical form and he/she can choose to apply the proposed schedules in the network via field technicians.

\subsection{Results}

The proposed MPC scheme has been operational for over 1 month with 1 hour sampling time and $H_{p}=24$. The calculation time, including running of demand forecaster and all steps described in section 3.5, was under 4 minutes on average and was never longer than 5 minutes. Since the control actions were not applied to a real system, the WDS which was actually controlled by local control loops did not behave as predicted by the MPC, see an example of real and predicted levels of the largest reservoir in this WDS illustrated in Figure 4.

Such mismatch between the real and predicted system behaviour makes the optimisation problem harder to solve, due to initial conditions generated via simulation of the the hydraulic model using the previously calculated control schedule and the most recent reservoir level measurements, as discussed in Section 3.5. If MPC predicts an increase in the reservoir level, while in fact the reservoir level decreases, such generated initial conditions may be infeasible due to violation of the reservoir level constraints. Furthermore, during the test period several field sensor failures occurred. As a result, CONOPT solver occasion- 


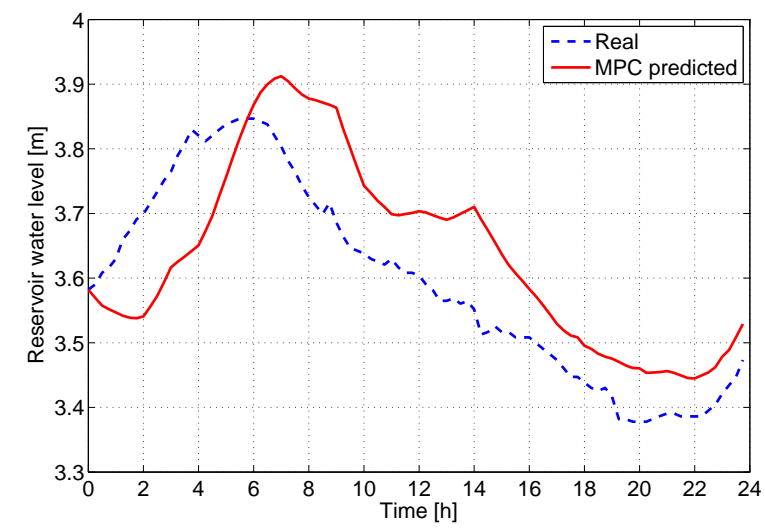

Fig. 4. Comparison of levels of the largest reservoir (RES4) in the WDS: Actual level from measurements and predicted level if the WDS was controlled by MPC.

ally converged at some local minima or failed to find a feasible solution; this happened in approximately 5\% of runs. In such cases some backup 'always working' control should be used, this however is beyond the scope of this paper.

In the Authors' earlier work the optimisation process employed in the proposed MPC scheme was used in an off-line manner on the same case study, see (Skworcow et al., 2009b). During off-line studies it has been observed that the operation of pumps and valves calculated by the optimisation process resulted in (compared to the actual operation): more intensive pumping during the cheap tariff period, use of larger reservoir volume, running pumps at lower speed and closer to their highest efficiency points.

In the off-line simulation study an average cost of electrical energy when the WDS is controlled by local loops was 402 units $^{1}$ per day. The data describing an actual pump operation during the on-line tests considered in this paper has not yet been made available to the Authors. However, during the on-line test period the demands and exports were the same or higher compared to the off-line study; hence, it can be assumed that the actual cost during the on-line test period was not smaller than the cost calculated during the off-line study. Histogram of a predicted cost if the system was actually controlled by MPC, calculated at each sampling interval using simulation, is illustrated in Figure 5. It can be observed that: (i) there is a potential for significant savings - approximately $30 \%$ and (ii) the bars indicating the cost between 340 and 400 units are likely to be a result of the optimisation process converging at some local minima.

\section{CONCLUSIONS}

This paper considered development of a methodology for on-line energy and leakage management in water distribution systems, formulated within model predictive control framework. The approach involves calculation of control actions, i.e. time schedules for pumps, valves and sources, to minimize the costs associated with energy used for water pumping and treatment and water losses due to leakage,

1 Actual cost in GBP cannot be revealed due to confidentiality agreement

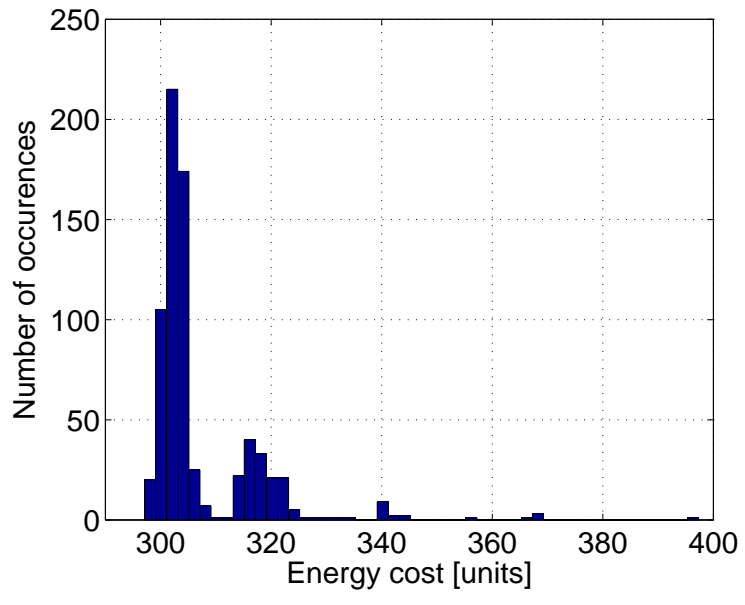

Fig. 5. MPC implementation scheme.

whilst satisfying all operational constraints. The method uses hydraulic models of WDS and enables adaptation to structural changes in a network via inclusion of an automatic model reduction module.

An on-line implementation of the proposed control scheme employed an open-source hydraulic simulator called EPAnet, mathematical modelling language called GAMS and a nonlinear programming solver called CONOPT. Subsequently it has been integrated with an industrial SCADA system provided by $\mathrm{ABB}$ and interfaced with a medium-scale WDS being part of Yorkshire Water Services. The scheme is currently being tested using on-line data albeit without the control actions being actually applied in the WDS. Preliminary results indicate a potential for significant savings in the cost of electrical energy, compared to current network operation.

\section{ACKNOWLEDGEMENTS}

The Authors are grateful to ABB Germany for their help in implementation and testing of the control scheme described in this paper.

\section{REFERENCES}

Biscos, C., Mulholland, M., Lann, M.L., Buckley, C., and Brouckaert, C. (2003). Optimal operation of water distribution networks by predictive control using MINLP. Water SA, 29, 393-404.

Bounds, P., Kahler, J., and Ulanicki, B. (2006). Efficient energy management of a large-scale water supply system. Civil Engineering and Environmental Systems, 23(3), $209-220$

Brdys, M. and Ulanicki, B. (1994). Water Systems: Structures, Algorithms and Applications. Prentice Hall UK.

Brooke, A., Kendrick, D., Meeraus, A., and Raman, R. (1998). GAMS: A user's guide. GAMS Development Corporation, Washington, USA.

Bunn, S. (2007). Greenhouse gas reduction as an additional benefit of optimal pump scheduling for water utilities. Proceedings of the Water Environment Federation, 2007, 1243-1252.

Camacho, E. and Bordons, C. (2004). Model predictive control. Springer-Verlag. 
Drud, A. (1985). Conopt: A grg code for large sparse dynamic non-linear optimisation problems. Mathematical Programming, 31, 153-191.

Igreja, J.M. and Lemos, J.M. (2009). Nonlinear Model Predictive Control, volume 84/2009 of Lecture Notes in Control and Information Sciences, chapter Nonlinear Model Predictive Control of a Water Distribution Canal Pool, 521-529. Springer Berlin / Heidelberg.

Morari, M. and Lee, J. (1999). Model predictive control: past, present and future. Computers and Chemical Engineering, 23, 667-682.

Ormsbee, L. and Lansey, K. (2007). Optimal control in water-supply pumping system. Journal of Water Resources Planning and Management, 120(2), 237-252.

Overloop, P.V. (2006). Model Predictive Control on Open Water Systems. IOS Press.

Qin, S. and Badgwell, T. (2003). A survey of industrial model predictive control technology. Control Engineering Practice, 11, 733-764.

Rance, J., Coulbeck, B., Kosov, S., Bounds, P., and Ulanicki, B. (2001). Finesse - a comprehensive software package for water network modelling and optimisation. In Water Software Systems: Theory and Applications. Research Studies Press LTD, Baldock, England.

Skworcow, P., AbdelMeguid, H., Ulanicki, B., and Bounds, P. (2009a). Optimal pump scheduling with pressure control aspects: Case studies. In Integrating Water Systems: Proceedings of the 10th International Conference on Computing and Control in the Water Industry.

Skworcow, P., AbdelMeguid, H., Ulanicki, B., Bounds, P., and Patel, R. (2009b). Combined energy and pressure management in water distribution systems. In 11th $\mathrm{Wa}$ ter Distribution Systems Analysis Symposium, Kansas City, USA.

Ulanicki, B., Bounds, P., Rance, J., and Reynolds, L. (2000). Open and closed loop pressure control for leakage reduction. Urban Water, 2(2), 105-114.

Ulanicki, B., Kahler, J., and Coulbeck, B. (2008). Modeling the efficiency and power characteristics of a pump group. Journal of Water Resources Planning and Management, 134(1), 88-93.

Ulanicki, B., Kahler, J., and See, H. (2007). Dynamic optimization approach for solving an optimal scheduling problem in water distribution systems. Journal of Water Resources Planning and Management, 133(1), 23-32.

Ulanicki, B., Zehnpfund, A., and Martinez, F. (1996). Simplification of water network models. In A. Muller (ed.), Hydroinformatics 1996: Proceedings of the 2nd International Conference on Hydroinformatics, Switzerland, volume 2, 493-500. 